\title{
MRI Brain Image Enhancement Using XILINX System Generator and DWT
}

\author{
Gouri B. Deshpande and Dr.K. Ramesha
}

\begin{abstract}
This paper presents a novel method of enhancing the image quality of human brain obtained from Magnetic Resonance Imaging scans. Here, we are trying to enhance the brain image using Xilinx System Generator, which is a DSP tool devised by Xilinx Corporation. The architecture presented in this paper utilizes a graphical user interface that bands together MATLAB Simulink and XSG offering appropriate hardware implementation. Hence, the quality of image is improved. These images are, then, fed to artificial neural network, for normality or abnormality classification of brain. This paper utilizes ANN using multiperceptron and back propagation method. This method first makes use of discrete wavelet transform to extract energy values from enhanced images using filters and then it is fed to ANN for further classification. The image enhancement using $X S G$, has its performance measured in terms of resource utilization by using FPGA XC5VLX50T is also measured.
\end{abstract}

Keywords--- Artificial Neural Network (ANN), Field Programmable Gate Array (FPGA), Image Enhancement, Magnetic Resonance Imaging (MRI), Xilinx System Generator (XSG)

\section{INTRODUCTION}

$\mathrm{D}$ IGITAL image processing is expanding in all the spheres of our life. It may be defense, aerospace, biomedical application, biometric field, space exploration. The management and usage of such digitally processed image is growing progressively. With a raise in the demand for better visual content, the technology needs to be renewed and modernized.

Image processing in digital means is required for proper analysis, inference and based on that apt decision making. Hence, the image content and quality should be improvised. In this paper, we try to stress on processing the image in terms of pixels and work out an algorithm for these pixels present in the image and reconstruct the image, back from the pixel values.

In this paper, we are trying to enhance the quality and Visual content of MRI of the brain using Xilinx System and classifying further for the brain image is normal or abnormal. Quality of image can be made better in many ways. Here, we are just trying to enhance the image for better perception and

Gouri B. Deshpande, M. Tech Scholar, Department of Electronics and Communication Engineering, Dr. Ambedkar Institute of Technology, Bengaluru, India. E-mail:gourideshpande391@yahoo.com .

Dr.K. Ramesha, Professor, Department of Electronics and Communication Engineering, Dr. Ambedkar Institute of Technology, Bengaluru, India.E-mail:kramesha13@gmail.com DOI: 10.9756/BIJAIP.8039 visual content. Also, we are providing hardware implementation on FPGA Virtex 5 making use of Xilinx system Generator. XSG is a versatile DSP tool developed by Xilinx Corporation.

Magnetic Resonance Imaging (MRI) is a medical imaging technique which produces high quality resolution images of anatomical parts like brain, chest etc. This technique is used in areas like biomedical field and provides critical information for clinical diagnosis and research.

FPGA is beneficial over DSP or ASIC based hardware implementation as it has higher reprogram ability, parallel computational ability. FPGA also provides a straightforward approach while implementing it by using Verilog Hardware Descriptive Language.

In this paper, we aim to implement an algorithm for image enhancement on brain MRI image using system generator. We start our process with implementing our architecture for enhancement using blocks available in Simulink reservoir of MATLAB R2012a and .m files. Using ISE 13.4 tool from Xilinx, which is compatible with System generator, we obtain a bitstream code. Also again by using Xilinx simulator we can simulate the results on modelsim. Using Chipscope pro tool, we dump the bitstream file on FPGA for hardware The design and implementation on FPGA hardware is done using MATLAB based Simulink blocks in XSG prototype.

\section{LITERATURE SURVEY}

An extensive research has been performed by the researchers on image enhancement and classification based on ANN. In the method proposed by S. Allin Christe et al., [1] uses edge detection technique. Many image filtering algorithms have been proposed and tumor characterization is done using XSG, where they achieve resource utilization up to $50 \%$ for hardware implementation.

Kalyani Dakre and P.N Pusdekar [2] try to focus on the different image enhancement algorithms like contrast stretching, thresholding, negative transformation etc., to be implemented on FPGA using XSG. Zhang Shanshan and Wiang Zong [3] have proposed architecture for recognizing features of edges in a vehicle. The system implementation is using hardware Spartan 3e to get the resource utilization. In the paper presented by Pooja Gupta and Kuldip Pahwa [4] stress on how to improve the image visual quality using pixel reconstruction and Gabor filtering technique, the color enhancement showing its maximum improvement.

Arun Kumar Mittal and Sukhwinder Singh [5] explained brain tumor detection can be made, using histogram equalization and image processing techniques, which were used to segment the brain tumour region. In [6], the paper has 
implemented different image enhancement techniques using spatial domain and frequency domain to enhance the visual quality and have tried to implement it on FPGA.

Image enhancement methods approach using Verilog Hardware Descriptive Language by Iuliana Chiuchisan et al., [7] focuses on image enhancement in spatial domain for the point processes algorithms like contrast stretching threshold operation, to provide easier approach to implement it on hardware. K. Anil kumar and Vijay Kumar [8] have presented various image processing operations using FPGA. They have also utilized Matlab Simulink blocks and XSG.

Sami Hasan et al., [9] have presented parallel 2D image filtering algorithm and its implementation on FPGA. All total of nine algorithms have been implemented using Xilinx System generator within 12.3 Design suite and verified the results using two virtex 6 FPGA board. Tarek M.Bittibissi et al., [10] have implemented five image processing algorithms in spatial domain like median filter, contrast stretching, Histogram equalization etc. and implemented it on FPGA Cyclon III under Altera systems.

A.C Suthar and et al., [11] have presented hardware software co-simulation which can be done in model based approach and about enhancement of the image. The paper presented by Menaka R and Rohini S [12], is dealing with detection of blockage of arteries in human brain by using watershed transform approach. Their neural network implementation has an efficiency of $90 \%$ and hardware implementation is done on Spartan 3E.

In [13], the authors Yousuf Khan and et al., have used edge detection on Vascular images. They have enhanced the images using simulink model. Ed-Edily et al., [14] have proposed an automatic tumor detection and localization in MRI images which can detect and locate the tumor using edge detection and segmentation methods.

Y. Zang et al., [15] have presented an automated and accurate classification of MR brain images using adaptive chaotic swarm optimization method. They have used Feed forward network to classify the image as normal or abnormal. This method first employs a wavelet transform to extract features from images, and then applies the technique of principle component analysis (PCA) to reduce the dimensions of features. The reduced features are sent to an FNN, and these parameters are optimized.

In [16], Alareqi Mohamed et al., have presented hardware software co-simulation for image processing algorithms using system generator. In [17], V. Elaraman and G.Rajkumar have presented the paper on Implementing point processes on FPGA. They have utilised system generator to provide hardware in the loop and cosimulation approach.

Aniket Ingle and Vrushali G. Raut [18] developed an algorithm for edge detection using Xilinx system generator and they have hardware implementation on Spartan 3e. Kavitkar S.G and Paikrao P.L [19] have implemented many edge detection algorithms such as Roberts edge detection, Sobel edge detection etc. using Xilinx system Generator. They have also provided FPGA implementation to the generated algorithms to provide hardware in the loop approach.

\section{PROPOSED MODEL}

The main block diagram for implementation of the system is shown in Figure 1. The proposed block diagram for hardware implementation on FPGA using Xilinx system generator is shown in Figure 2.

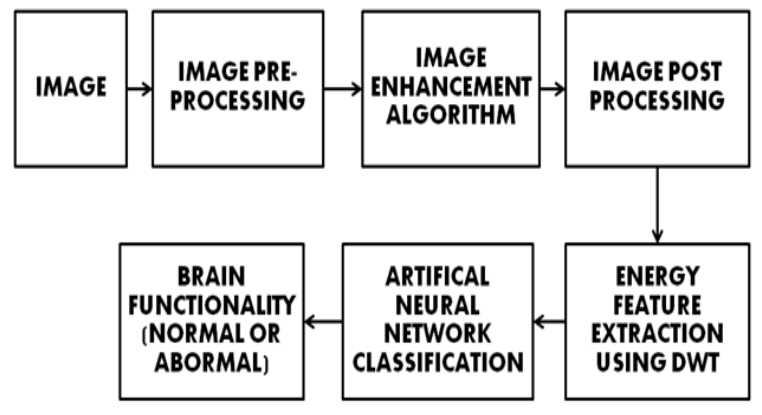

Fig. 1: Block Diagram of the System

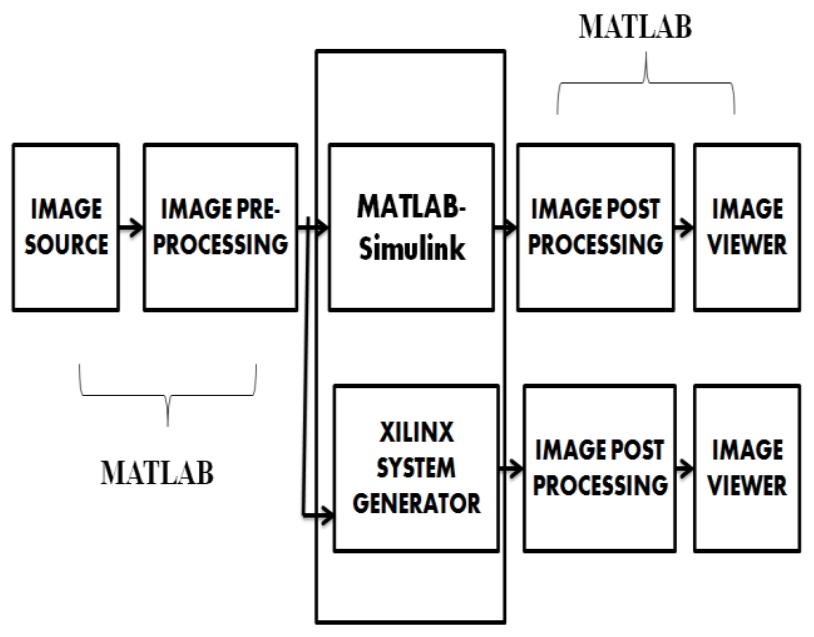

Fig. 2: Design Flow for Hardware Implementation

\section{A. Image Pre-Processing Block}

Any image processing will have pre-processing block present always. This is a common block which suppresses the unwanted noise present in the image required for further processing. The other name for image preprocessing is as filtration. Image preprocessing has been done here, to remove the noise present in the image such as speckle noise, foreground noise, background noise etc. present in the image. Due to preprocessing, the input to FPGA is in the form of test vector array which is suitable bit stream compilation using Xilinx for FPGA using the system generator. The image preprocessing blocks shown in Figure 3 contains the following:

- Resize: By setting up an input dimension for an image and bi-cubic interpolation, it helps in preserving finer details of an image.

- Convert 2D to 1D: converts two dimensional pixels present in an image to one dimensional or single dimensional array of pixel values.

- Frame conversion and buffer: helps in setting the sampling mode and buffer helps in storage of pixel values. 


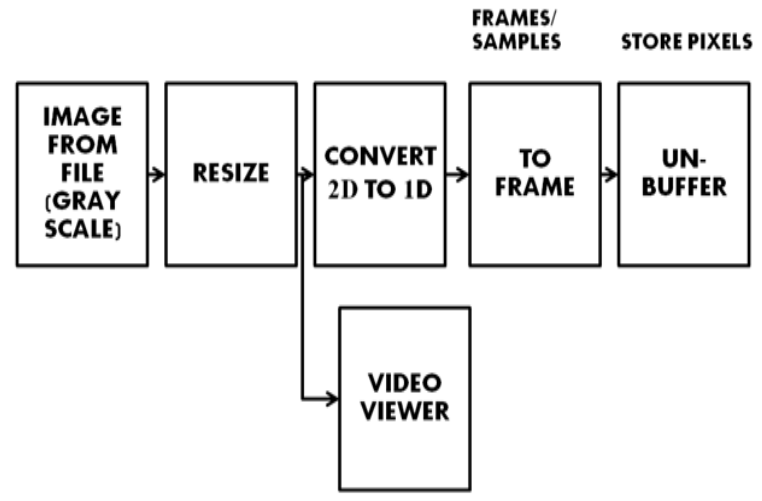

Fig. 3: Image Pre-processing Block

\section{B. Image Enhancement Block}

This is the most important block. Here, the image enhancement block, we are trying to increase the brightness and contrast of the brain MRI image. Here, we are using Gabor filtering and histogram equalization method to enhance the image. There is an automatic generation of Verilog code from MATLAB-Simulink blocks present in the algorithm. For image enhancement, we are trying to use two techniques:

\section{Histogram Equalization}

Histogram equalization is one of the technique used in spatial domain. Due to the simplicity and performance on all kinds of images, histogram equalization is in wide use. Histogram equalization technique performs contrast stretching on the neighborhood pixels in the image. If there are $\mathrm{m}$ different pixel values such that $\mathrm{m} \geq 0$ having $\mathrm{L}$ gray levels $\mathrm{xi}$ where $\mathrm{i}=0,1,2 \ldots \mathrm{L}-1$, with probability $p_{u}\left(x_{i}\right)$, then probability $\mathrm{p}\left(\mathrm{x}_{\mathrm{i}}\right)$ can be calculated from histogram $\mathrm{h}\left(\mathrm{x}_{\mathrm{i}}\right)$, the number of pixels with value $x_{i}$

$$
\mathrm{pu}(\mathrm{xi})=\mathrm{h}(\mathrm{xi}) / \sum_{\mathrm{i}=0}^{\mathrm{L}-1} \mathrm{~h}(\mathrm{xi})----(1)
$$

\section{Gabor Filtering}

The frequency and orientation representation of Gabor filter in spatial domain is similar to that of the human eye. The Gabor filter is a Gaussian function modulated by sinusoidal wave. The Gabor filter is given in the Equation (2)

$$
\mathrm{h}(\mathrm{x}, \mathrm{y})=\mathrm{g}(\mathrm{x}, \mathrm{y}) * \mathrm{~s}(\mathrm{x}, \mathrm{y})
$$

where $\mathrm{g}(\mathrm{x}, \mathrm{y})$ is Gaussian function ;

$\mathrm{s}(\mathrm{x}, \mathrm{y})$ is sinusoidal function.

By the convolution property of the Gabor filter, the convolution of Fourier transforms of Gaussian and Harmonic function gives the impulse response of Gabor function.

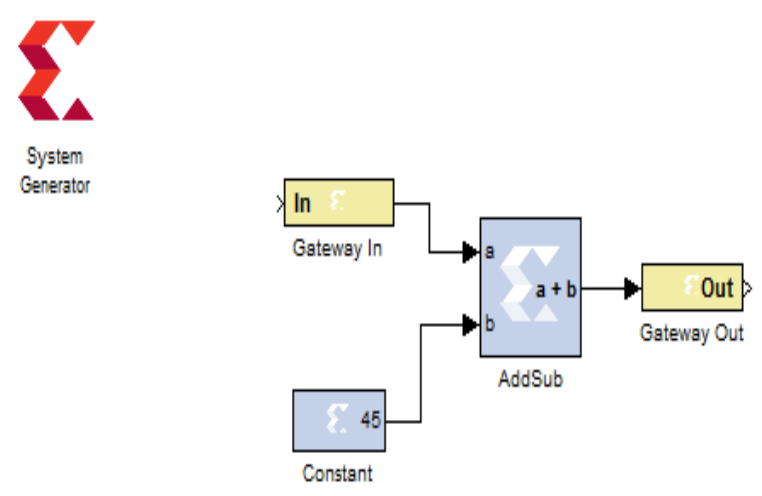

Fig. 4: Image Enhancement Block

\section{Image Post Processing}

Image post processing is the converse of the image preprocessing block and plays a different role. Image post processing helps in recreation of the image from single array of pixels i.e. displaying back the whole image. The image post processing block shown in Figure 5 contains:

- Data conversion type: This block converts the image into unsigned integer UINT format which is of 8 bits.

- Buffer: It is a storage device; used for storing the pixel values. It also converts frames to samples at a lower conversion rate.

- Convert 1D to 2D: converts single array of pixels to two dimensional array image matrix.

- Video Viewer: It displays the output image on the screen.

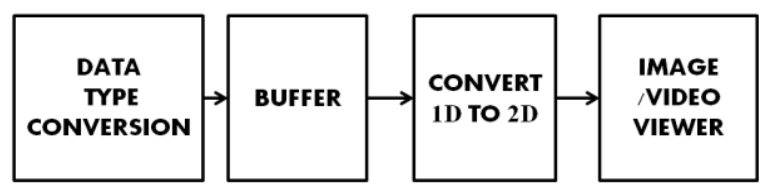

Fig. 5: Image Post processing Block

\section{Energy Feature Extraction using DWT}

Once we get the enhanced image, we try extracting energy feature values using DWT, to get the image in all the four directions i.e. 0, 90, 180, 270 degrees. For this, we are dealing with following types of filters:

1. Daubechies filter- 2 to 24 .

2. Biorthogonal filter- 3.2 to 7.9 .

3. Symlets filter- 2.2 to 23 .

The above DWT filters are characterized by number of vanishing moments. As the vanishing value increases, the sharpness of the curve increases. The DWT is applied for two levels.

\section{Bi-orthogonal Filter}

This filter may be orthogonal or non orthogonal. This is an invertible filter. It can be used to generate symmetric wavelet function. In this paper, we are using biorthogonal filter -3.3, 3.5, 3.7. 


\section{Symlets Filter}

They are modified version of Daubechies wavelets with more symmetricity. They range from 2 to 23 in which we are using sym3.

\section{Daubechies Filter}

Its an orthogonal wavelet having more number of vanishing moments. We are using Daubechies filter3.

\section{E. Artificial Neural Network Classification}

The artificial neural network is used to classify the normality or abnormality of the brain. The abnormality of brain image is classified using technique called multilayer perceptron using back propagation technique.

A multilayer perceptron (MLP) is a feed-forward artificial neural network model that maps sets of input data onto a set of appropriate outputs. A MLP consists of multiple layers of nodes in a directed graph, with each layer fully connected to the next one. Except for the input nodes, each node is a neuron (or processing element) with a nonlinear activation function.

MLP utilizes a technique called back-propagation for training the network. Back-propagation or "backward propagation of errors", is a common method of training artificial neural networks used in conjunction with an optimization method such as gradient descent. Backpropagation requires a known, desired output for each input value in order to calculate the loss function gradient.

\section{Algorithm}

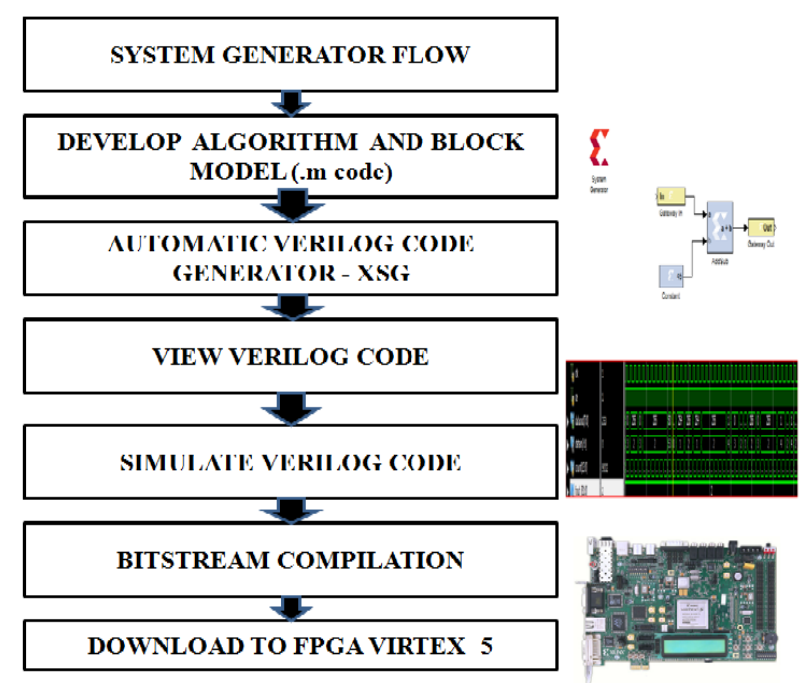

Fig. 6: Algorithm for Image Enhancement

The algorithm is shown in Figure 6. First using Simulink, we implement our architecture for enhancement which is a block model in MATLAB R2012a with an extension .m files. Using ISE 13.4 tool from Xilinx, which is compatible with System generator, we obtain a bit stream code. Also again by using Xilinx simulator we can simulate the results on Modelsim simulator. Using Chipscope pro tool, we dump the bit stream file on FPGA for hardware. The design and implementation on FPGA hardware is done using MATLAB based Simulink blocks in XSG prototype.

\section{PERFORMANCE ANALYSIS}

The design and implemented image enhancement algorithm for the MRI image is shown in Figure 7.

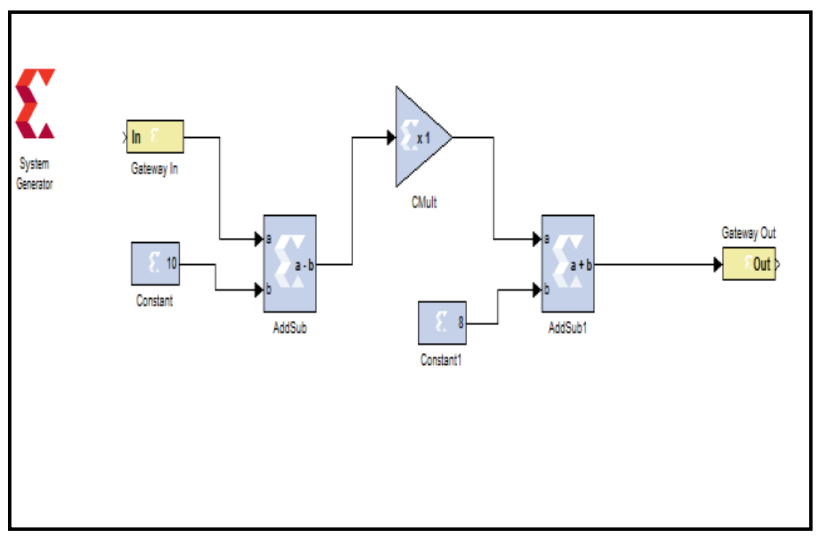

Fig. 7: Image Enhancement Algorithm

The RTL schematic view for the proposed image enhancement algorithm is shown in Figure 8.

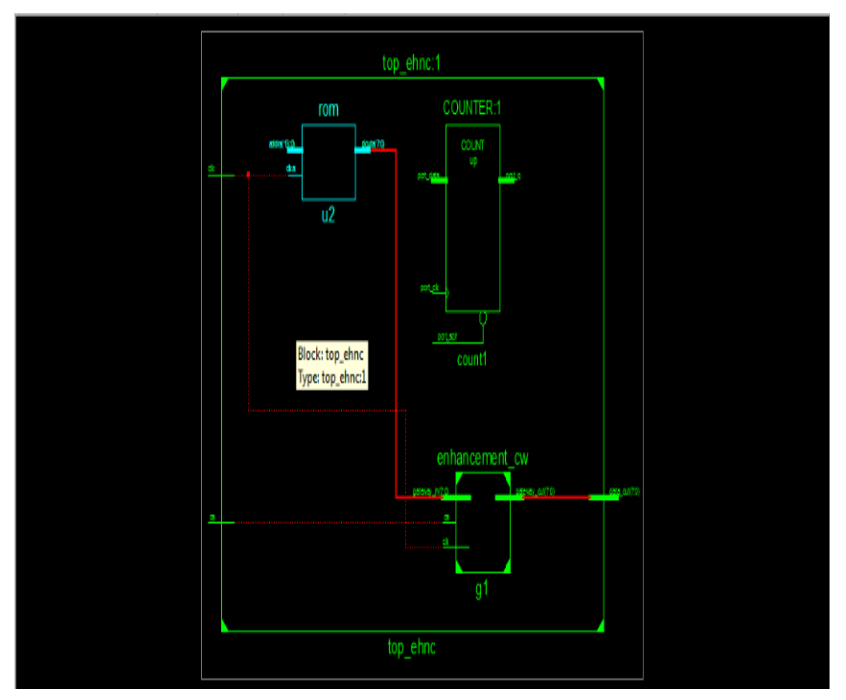

Fig. 8: RTL Schematic View

The enhanced image output for a given MR brain image is shown in Figure 9 and Figure 10.

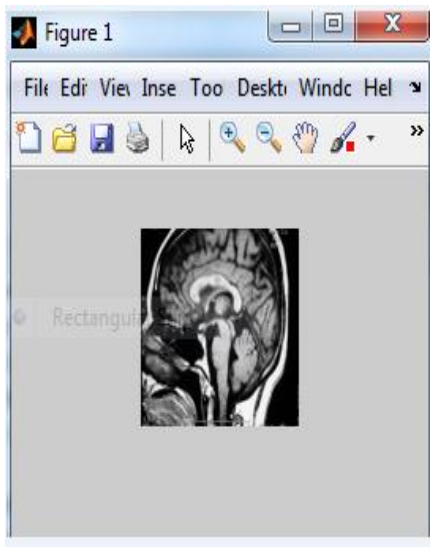

Fig. 9: Input Image

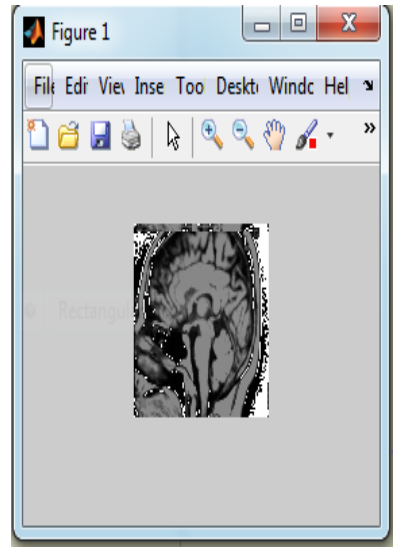

Output Image 


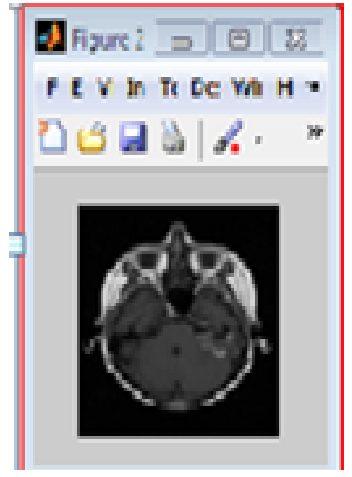

Fig. 10: Input Image

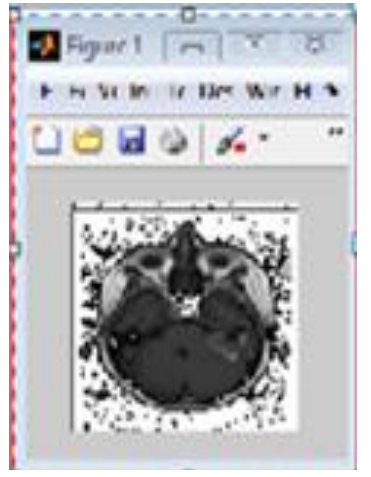

Output Image
The mapping of pixel values on the output as generated in the simulation results by Xilinx ISE 13.4 is shown in Figure 11 for the brain image shown in Figure 9.

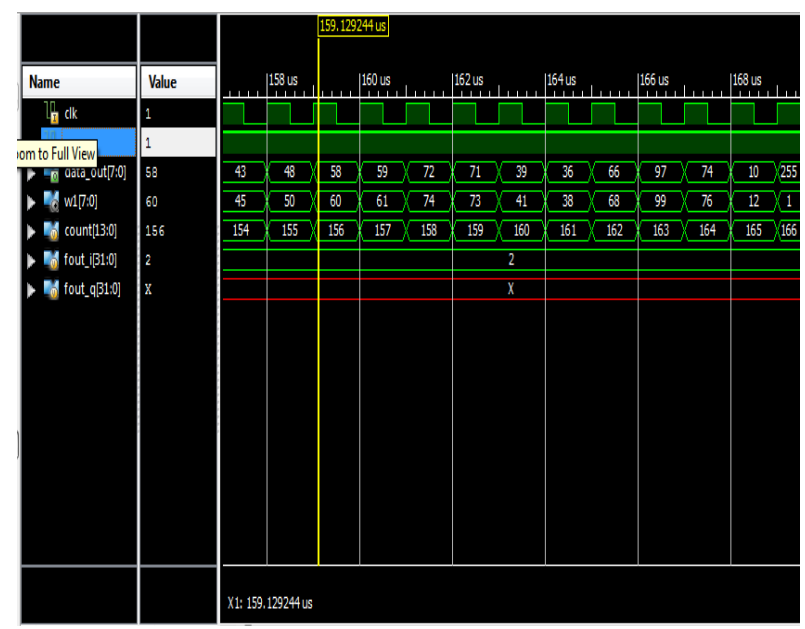

Fig. 11: Simulation Results of Image shown in Figure 8

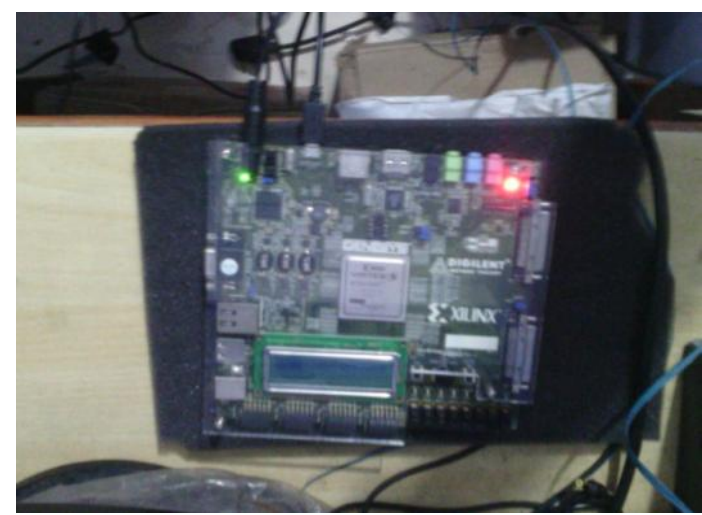

Fig. 12: Virtex 5 FPGA kit

The hardware implementation on FPGA virtex 5 is shown in Figure 12. We are using XILINX ISE 13.4 and Matlab R2012a.The enhanced image is given to artificial neural network to extract energy feature values using DWT filters for Figure 8 is shown in Figure 13,14,15,16,17.

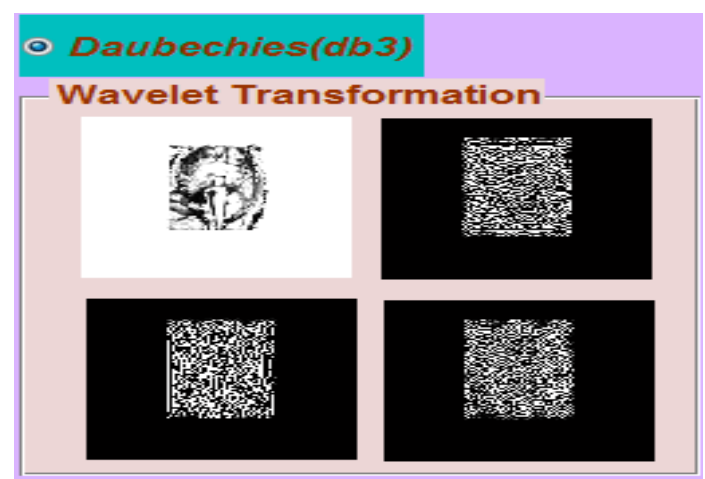

Fig. 13: Daubechies Type3 Filter Output

Table 1: Resource Utilization in Hardware Implementation

\begin{tabular}{|l|c|c|c|c|c|}
\hline RESOURCES & AVAILABLE & USED & $\begin{array}{l}\text { PROPOSED METHOD } \\
\text { RESOURCES ULITISATION } \\
(\%)\end{array}$ & $\begin{array}{l}\text { RESOURCE UTILISATION } \\
\text { BY CHRISTE ET AL (\%) }\end{array}$ & $\begin{array}{l}\text { RESOURCE } \\
\text { UTILISATION BY } \\
\text { ZHANG(\%) }\end{array}$ \\
\hline FLIP FLOPS & 28800 & 87 & 1 & 2 & 4 \\
\hline SLICES & 28800 & 88 & 1 & 2.5 & 5 \\
\hline LUTS & 28800 & 120 & 1 & 1.5 & 3 \\
\hline
\end{tabular}

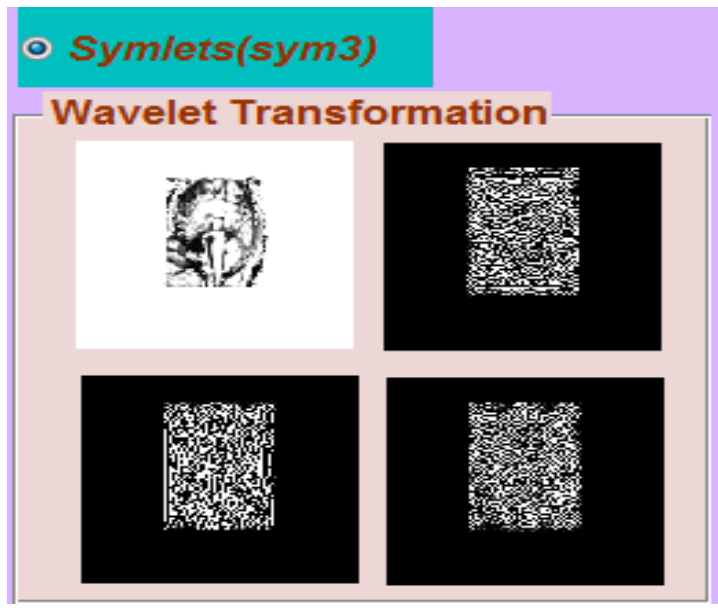

Fig. 14: Symlets Type 3 Filter Output

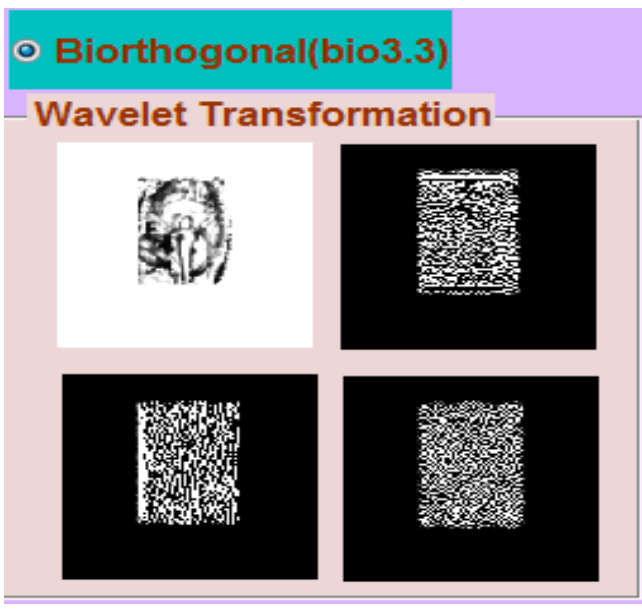

Fig. 15: Biorthogonal Type 3.3 Filter Output 


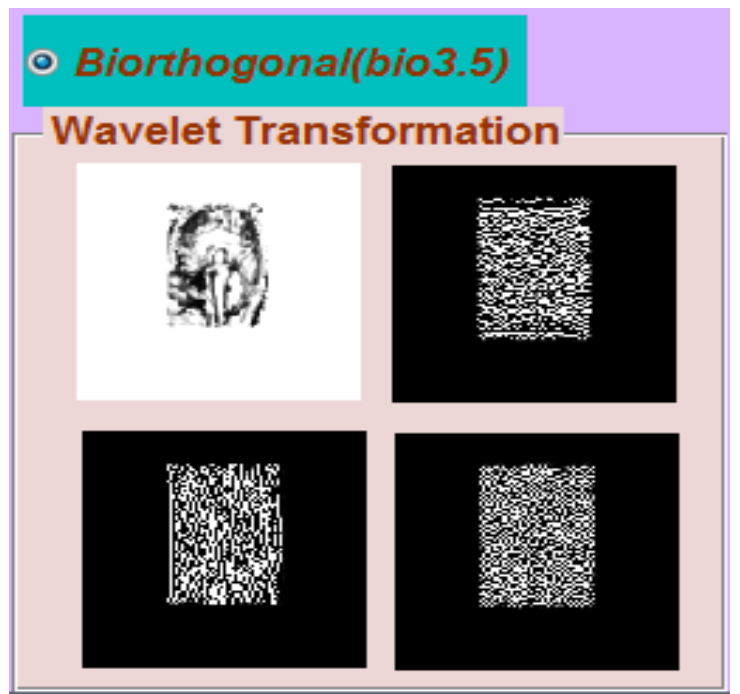

Fig. 16: Biorthogonal Type 3.5 Filter Output

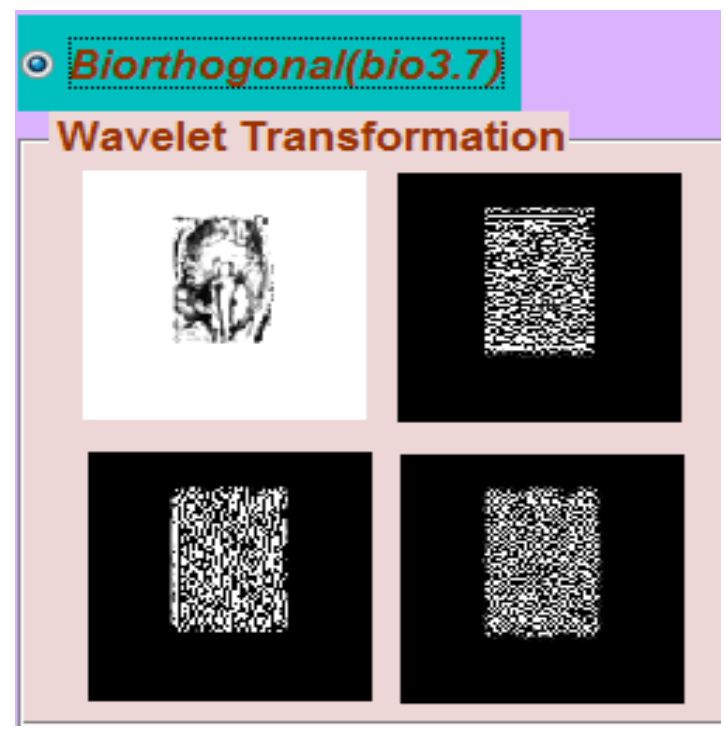

Fig. 17: Biorthogonal Filter 3.7 Output

Based on the obtained energy values from the filters the brain image can be declared as normal or abnormal.

\section{CONCLUSION}

Xilinx System Generator is a new versatile tool built to perform image processing in hardware and software. It provides rapid means to do hardware implementation of complex techniques used for processing images with minimum resource and minimum delay. Also, XSG provides parallelism, robustness and automatic area minimization. The need of rapid prototyping tools such as MATLAB Simulink and Xilinx System Generator are increasingly important in recent times because of time-to-constraints. It also provides simplicity and easier to implement on Hardware. The enhancement of MRI image can be further be helpful to the surgeons to operate on, at accurate locations of the brain. The early detection of the disease related to brain can be considered as the future scope of the work.

\section{REFERENCES}

[1] S.Allin Christe, M. Vignesh and A. Kandaswamy, "An Efficient FPGA Implementation of MRI Image Filtering and Tumor Characterization using Xilinx System Generator", International Journal of VLSI design \& Communication Systems, Vol 2, issue 4, pp. 95-109, December 2011.

[2] Kalyani A. Dakre and P. N. Pusdekar, " Review on Image Enhancement using Hardware co-simulation for Biomedical operations", International Journal of Advanced Research in Computer Engineering \& Technology, vol 3,issue 12, pp. 4438-4441, December 2014.

[3] Zhang Shanshan and Wang Xiaohong on "Vehicle Image Edge Detection Algorithm Hardware Implementation on FPGA", International Conference on Computer Application and System Modeling, vol.109, pp. 325-343,2010.

[4] Pooja Gupta and Kuldip Pahwa, “ Visual Quality Improvement of Medical Images using Pixel Reconstruction followed by Gabor enhancement technique", International Journal of Signal Processing, Image Processing and Pattern Recognition, vol 7, issue 6, pp. 297302,2014

[5] Arun Kumar Mittal and Sukhwinder Singh ,“ Brain Tumor Detection with Histogram Equalization and Morphological Image Processing Techniques", International Journal for Innovative Research in Science \& Technology, vol 1 issue 3, pp.28-31, 2014.

[6] Nirmala S.O, T.D. Dongale and R. K .Kamat, " Review on Image Enhancement techniques: FPGA implementation Perspective", International Journal of Electronics Communication and Computer Technology, vol 2, issue 6, pp. 270-274, November 2012.

[7] Iuliana Chiuchisan, Marius Cerlinca, Alin-Dan Potorac, and Adrian Graur," Image Enhancement Methods approach using Verilog Hardware Descriptive Language", 11th International Conference on Development and Application Systems, pp.144-148, May 17-19, 2012.

[8] K. Anil Kumar and M. Vijay Kumar, "Implementation of Image Processing Lab Using Xilinx System Generator", Advances in Image and Video Processing, vol 2,issue 5, pp.27-35, $25^{\text {th }}$ September 2014.

[9] Sami Hasan, Said Boussakta and Alex Yakovlev, "FPGA-Based Architecture for a Generalized Parallel 2-D MRI Filtering Algorithm", American Journal of Engineering and Applied Sciences, vol 4,issue 4, pp. 566-575, 2011.

[10] Tarek M. Bittibssi, Gouda I. Salama, Yehia Z. Mehaseb and Adel E. Henawy on "Image Enhancement Algorithms using FPGA", International Journal of Computer Science \& Communication Networks, vol 2,issue 4, pp. 536-542.

[11] A.C.Suthar, Mohammed Vayada, C.B.Patel, G.R.Kulkarni on "Hardware Software Co-simulation for Image Processing Applications", International Journal of Computer Science Issues, vol 9,issue 2., March 2012.

[12] Menaka R and Rohini. S, "Efficient Detection of Ischemic Stroke from MRI Images Using Wavelet Transform", International Journal of Computer Science and Information Technology Research, vol.2, issue 3, pp.446-454,2014.

[13] Mohammed Yousuf Khan, Masarath Nayeem Tayyaba, M.A.Raheem, Ayesha Siddiqua and Syed Sameena, " Image Enhancement and Hardware implementation of Edge Detected Vascular Images using Simulink Model" , International Journal of Advanced Research in Computer and Communication Engineering, vol 3, issue 4, pp.63856388, 2014.

[14] Ed-Edily Mohd., Azhari, Muhd. Mudzakkir Mohd. Hatta, Zaw Zaw Htike and Shoon Lei Win, "Brain Tumor Detection And Localization In Magnetic Resonance Imaging", International Journal of Information Technology Convergence and Services, vol 4, issue 1, February-2014.

[15] Y. Zhang, S. Wang, and $\mathrm{L}$. Wu, "A novel method for magnetic resonance brain image classification based on adaptive chaotic PSO", Progress In Electromagnetics Research, vol. 10,issue 9, pp.325-343, 2010.

[16] Alareqi Mohammed, Elgouri Rachid and Hlou Lamaari , " High Level FPGA modeling for Image Processing Algorithms Using Xilinx system Generator", International Journal of Computer Science and Telecommunications, vol 5, issue 6, pp.01-08,June 2014.

[17] V. Elaraman and G. Rajkumar , "FPGA implementation of Point Processes using Xilinx System Generator", Journal of Theoretical and Applied Information Technology, vol 21 issue 41, pp.201-206,July 2012.

[18] Aniket A. Ingle and Vrushali G. Raut, "Hardware Software Cosimulation of Edge Detection for Image Processing System using Delay 
Block in XSG", International Journal of Research in Engineering and Technology, vol. 03, issue 5,pp.549-553, May-2014.

[19] Kavitkar S. G and Paikrao P. L, "Hardware Implementation of Edge Detection Algorithm- A Review", International Journal of Engineering Sciences \& Research Technology, vol 3, issue 2, pp.755-759, February, 2014.

[20] Neha P. Raut and A. V Gokhale, "FPGA Implementation for Image Processing Algorithms Using Xilinx System Generator", IOSR Journal of VLSI and Signal Processing, vol 2, issue 4, pp. 26-36, May-June 2013.

[21] Anil .K Jain, "Fundamentals of Digital Image Processing", Pearson Prentice Hall, 2005.

[22] Rafael C.Gonzales and Richard. E Woods, "Digital Image Processing", Prentice Hall, 2004.

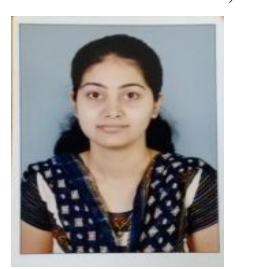

Gouri B. Deshpande is currently pursuing her M.Tech in VLSI Design and Embedded Systems, Dr. Ambedkar Institute Technology, Bengaluru. She completed her B.E. in Electronics and Communication from A.I.E.T, Gulbarga. Her interests include Image Processing, VLSI designing and Communication engineering.

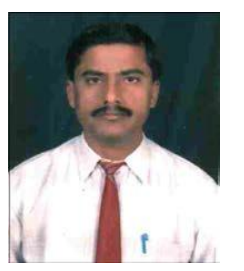

Ramesha K. was awarded B.E. degree in E \& C from Gulbarga University and M.Tech degree in Electronics from Visvesvaraya Technological University. He pursued his Ph.D from JNTU, Hyderabad. He has over 14 research publications in referred International Journals and Conference Proceedings. He is currently working as Professor in the Department of Electronics and Communication Engineering, Dr. Ambedkar Institute of Technology, Bengaluru. His research interest includes Image Processing, Biometrics, Pattern recognition and Communication Engineering. 